\title{
Mapping Ephemeral Stream Networks in Desert Environments Using Very- High-Spatial-Resolution Multispectral Remote Sensing
}

\author{
Yuki Hamada, ${ }^{1}{ }^{1}$ Ben L. O’Connor, ${ }^{2}$ Andrew B. Orr, ${ }^{1}$ and Kelsey K. Wuthrich ${ }^{1}$
}

\begin{abstract}
Mapping of ephemeral streams in desert environments is crucial to understanding the impacts to hydrologic and ecosystem functions caused by land-use changes. Available mapping methods at the watershed-scale typically underestimate total channel length and the size of channel networks. Although remote sensing is effective for obtaining information on large areas, conventional techniques are often ineffective or cost-prohibitive for complex stream networks in expansive desert regions. Using very-high-spatial-resolution imagery, we developed a new algorithm to map desert ephemeral streams in the southwestern U.S., where utility-scale solar energy development is altering the landscape. Knowledge about landscape features such as shrubs and desert pavement and their spatial arrangement was integrated into the algorithm using spectral transformation and spatial statistical operations. The algorithm extracted ephemeral stream lengths approximately $900 \%$ greater than those identified in the National Hydrography Dataset. The accuracy in mapping channel areas and centerlines was as high as $92 \%$ and $91 \%$, respectively. Although the algorithm captured detailed stream channels, it often underestimated channels obscured by bright soils and sparse vegetation. Although further improvement is warranted, the algorithm provides an effective means of obtaining detailed information about ephemeral streams, which could make a significant contribution toward improving the hydrological modeling of desert environments.
\end{abstract}

Keywords: ephemeral streams; desert regions; very high resolution

${ }^{1}$ Environmental Science Division, Argonne National Laboratory, Argonne, Illinois 60439, USA

2 Department of Civil and Materials Engineering, University of Illinois at Chicago, Chicago, Illinois 60607, USA

* Corresponding author: Yuki Hamada; yhamada@anl.gov; 9700 South Cass Avenue, EVS-240, Argonne, Illinois 60439, USA; 630-252-0087 


\section{Introduction}

Water is the limiting factor for various functions in desert environments, and changes in water regimes affect various ecosystem services (Belnap 1995). In desert environments, water from infrequent rainfall is conveyed primarily by ephemeral streams; this water delivery and storage network is important with regard to several ecosystem functions, such as the establishment of critical habitats, groundwater recharge, nutrient and organic material transport, and ecological connectivity (Levick et al. 2008; Steward et al. 2012). Development in desert regions often results in the loss and degradation of ephemeral streams (Abella 2010; Belnap 1995; Jacobson and Jacobson 2013). Surface grading, soil compaction, and the removal of vegetation degrade ephemeral streams directly, as natural channels are altered, removed, or replaced by large stormwater conveyance channels, or indirectly, as a result of altered runoff patterns from development sites (Chin and Gregory 2001; Field and Lichvar 2007; Duniway and Herrick 2011).

Desert regions of the southwestern United States are ideal for utility-scale solar energy development because of the high solar insolation levels that exist there. Solar energy zones (SEZs), where the U.S. Department of Interior Bureau of Land Management (BLM) has prioritized the development of utility-scale solar facilities, have been designated on approximately 1,153 $\mathrm{km}^{2}$ of public lands across six states (BLM 2015). Federal agencies are expected to protect and manage the full array of resources and values on their managed lands, including hydrological and ecological resources. Given the vast and remote nature of the SEZs, cost-effective, repeatable, easily deployable methods for monitoring the land and natural resources within the footprint and vicinity of a solar development are needed to support sustainable, long-term monitoring of the potential impacts of solar energy projects. Especially important is the need for a cost-effective and reliable alternative approach for mapping finescale, detailed stream channel networks across desert landscapes.

Ephemeral streams are ubiquitous in desert environments, but they are typically not mapped, and their physical characteristics are usually not well known. These conditions make it difficult to quantify the ecosystem functions of ephemeral streams, and they limit the potential for monitoring alterations to ephemeral stream networks that result from development activities. The National Hydrography Dataset (NHD) is the primary resource for stream channel mapping information available in the U.S. (USGS 2008). Previous studies have shown that both the highresolution NHD (1:24,000 scale) and the medium-resolution NHD (1:100,000 scale) often underestimate the extent of headwater streams (Fritz et al. 2013), and that the classification (e.g., ephemeral, intermittent, or perennial) and mapping (i.e., positional accuracy) of these streams are often inaccurate (Caruso and Haynes 2011). Methods of generating synthetic stream channel networks from digital elevation models (DEMs) are available in several geographic information system (GIS) and hydrological modeling packages, but these methods are limited by the resolution of the DEM, which is typically at best on the order of $10 \mathrm{~m}$. This lack of stream channel identification and accuracy in location and extent limits the researchers' ability to assess hydrologic and ecosystem conditions at the local scale. The difficulty of accurately mapping and quantifying the critical functions of ephemeral streams at scales ranging from individual reaches to the watershed scale limits the development of best management practices, mitigation 
measures, and regulatory protection mechanisms that address land development impacts on ephemeral streams.

The physical characteristics of ephemeral streams in desert regions are largely controlled by climatic drivers and watershed characteristics such as elevation, slope, surficial soils, and parent geology (Pool 2005; Wohl et al. 2007). Unlike temperate stream channels, desert streams often exhibit a complex composition of both single-threaded and multi-threaded channels with compound cross-sections that do not exhibit consistent channel adjustments relative to hydrologic inputs (Tooth 2000; Vyverberg 2010). In desert landscapes, ephemeral stream channels exhibit small topographic gradients over large areas, and tributaries to larger dry washes are often considerably smaller and more abundant than for streams in less arid regions (Bull and Kirkby 2002). Some of these unique physical characteristics of desert ephemeral channels influence spectral properties of landscapes, imposing challenges in extracting stream channels through remote sensing methods. The absence of water considerably reduces the contrast between stream channels and surrounding land. In addition, surface materials in streambeds and uplands are often spectrally similar. Riparian vegetation in arid landscapes is often discontinuous and sparser and less vigorous than it is in temperate riparian habitat (Fisher 2006). These unique characteristics of arid stream channels suggest the need for methods for mapping ephemeral stream networks that are specific to desert regions.

Currently, the best methods for mapping ephemeral stream channel networks include field surveys and the manual digitization of channels from high-resolution imagery or topographic maps. However, these methods are impractical for covering large areas and for frequent mapping efforts. Remote sensing provides an effective means of obtaining landscape information over large areas while compensating for the drawbacks of field-based methods. Light Detection and Ranging (LiDAR) technology has been widely used to map streams and drainages. DEMs derived from LiDAR data provide vertical or height information to characterize depressions, compute flow direction, and link streams in a range of landscapes (Jenson 1991; Li and Wang 2010). LiDAR data have been used with a wavelet-based filtering technique (Lashermes et al. 2007), mathematical morphological and geodesic operators (Cho et al. 2011), and curvature analysis (Passalacqua et al. 2012) to map stream channels. The Automated Geospatial Watershed Assessment Tool (EPA 2014) also uses DEMs to parameterize streams within the model. Despite these advanced methods, the frequent use of LiDAR to produce detailed stream network maps would incur a high operational cost associated with large data volume and processing time. This cost would be important to consider when mapping ephemeral streams across an area repeatedly over the course of many years, as well as when the objectives include multiple land resource types. These issues indicate that an inexpensive alternative approach is needed to map fine-scale stream channel networks.

An image-based approach that uses traditional optical remote sensing represents an effective alternative for mapping detailed stream channel networks while overcoming the shortcomings of LiDAR data. The effectiveness of 30-m-resolution Landsat Thematic Mapper imagery, among the most widely used satellite image data, was tested for mapping complex channel networks (a braided river network in the Brahmaputra River and a tidal river network in the Berau Bay) in conjunction with a multiscale classification approach (Yang et al. 2014). This study indicated that spectral mixture within a pixel was one of the causes of overclassification of 
streams. The authors also noted that the delineation of small channels remained a major challenge. In a separate study, WorldView-2 multispectral imagery with 50-cm resolution was used to map supraglacial streams over the Greenland ice sheet (Yang and Smith 2013). Supraglacial stream features exhibit spectral reflectance signatures similar to those of surrounding landscape features. Despite the presence of stream water in supraglacial stream features, the subtle spectral contrast between channels and non-channel features constituted a challenge similar to that of extracting ephemeral channel networks in desert regions.

Very-high-spatial-resolution (VHSR) multispectral imagery resolves fine-scale features and phenomena in the landscape and reduces the level of mixed spectral reflectance observed within pixels in coarse- and moderate-resolution imagery. Spectral reflectance data from VHSR multispectral imagery provide information about the composition of the land surface and the abundance of the components on the ground. This information can be used to map various landscape features (e.g., vegetation, bright soil, desert pavement dry land). Many studies have shown the effectiveness of VHSR imagery in mapping land cover features and conditions found in arid environments. Hamada et al. (2013) mapped land surface components of shrublands (e.g., shrubs, herbaceous plants, and exposed soil) using 15-cm-resolution multispectral imagery with object-based image analysis. Witztum and Stow (2004) used surface brightness of the red spectral band of high-resolution imagery to examine soil compactness. The spatial patterns of vegetation and soil brightness can provide information that is useful in understanding the distributions of landscape features that may not be distinguishable solely on the basis of spectral reflectance values, such as dry desert stream channels.

New remote sensing methods for mapping ephemeral stream networks need to account for the unique characteristics of ephemeral stream channels in desert environments. Although many advanced techniques, such as machine-learning algorithms, have been successful in various mapping applications, they often do not fully integrate key landscape features and structures (Sant et al. 2014; Wang et al. 2014). The goal of this study was to develop a costeffective remote sensing methodology for mapping ephemeral stream networks in a desert landscape by using VHSR multispectral imagery. By using these data and incorporating knowledge about desert landscapes into the ephemeral channel mapping algorithm, we developed a method to cost-effectively characterize land-based resources that are of concern to resource managers.

\section{Materials and Methods}

\subsection{Study Area}

The study area is located in the Palo Verde Mesa in eastern Riverside County, California (Figure 1). The area is a part of the Riverside East SEZ, which is the largest of the SEZs designated by the BLM (BLM and DOE 2012). The total area designated for utility-scale solar energy projects within this SEZ is $599 \mathrm{~km}^{2}$. As of September 2014, four solar projects had been authorized, and three project applications were pending. These proposed projects cover an area equal to about $21 \%$ of the SEZ area. 


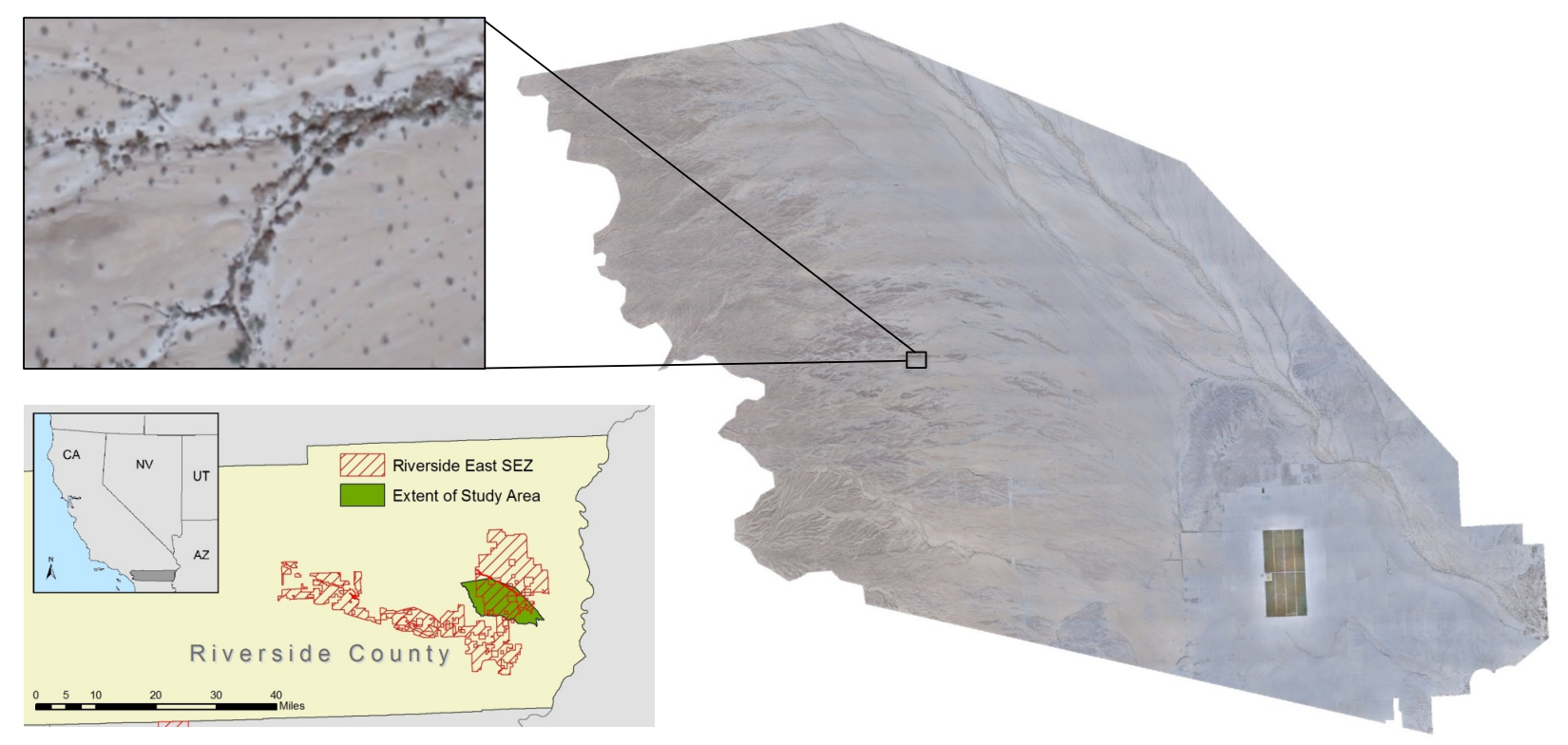

Figure 1. Study area. Irrigated fields are visible in the southeast corner of the study area. McCoy Wash, which runs in a northwest-southeast direction, is visible running parallel to the northeast boundary of the study area. The upper left hand inset image shows a close-up view of the ephemeral channels.

The study area comprises approximately $150 \mathrm{~km}^{2}$ and contains one solar energy project area. The area is covered by sparsely distributed vegetation, consisting primarily of desert scrub species (e.g., creosotebush [Larrea tridentata] and white bursage [Ambrosia dumosa]), microphyll trees (e.g., blue palo verde [Parkinsonia florida] and ironwood [Olneya tesota]), and annual herbs. The area exhibits typical desert stream networks made up of single-thread and braided channels mixed with discontinuous forms. The ground surface consists mainly of erodible soils formed by silt and sand, desert pavement, and sparsely distributed biological soil crust. Approximately a quarter of the area to the west exhibits rugged topography; there is a gain in elevation of about $130 \mathrm{~m}$ (from 185 to $315 \mathrm{~m}$ above mean sea level). The rest of the study area has a subtle topographic gradient toward the foothills. The annual precipitation in the area is $9.7 \mathrm{~cm}$, and average minimum and maximum temperatures are $38^{\circ} \mathrm{F}$ and $109^{\circ} \mathrm{F}$, respectively.

\subsection{Data}

VHSR multispectral images of the study area were collected on November 12 and 13, 2012, by using a fixed-wing aircraft flying about 1,350 $\mathrm{m}$ above the ground. The VHSR images consist of four spectral bands within the visible and near-infrared (NIR) spectral regions. The images have a 15-cm spatial resolution and an 8-bit data range. A total of 1,527 frames were mosaicked and clipped to the study area. ASD FieldSpec Pro (http://www.asdi.com) was used to collect spectral reflectance values within the visible and NIR spectral regions (325-1,075 nm) from bright, sandy soils as a white reference target in order to calibrate image pixel values to 
relative reflectance values. Six 50- to 100-m-long ephemeral stream segments were recorded during the field surveys on September 25 and 26, 2012. Plant life-form types and canopy diameters were recorded from 32 locations along the six ephemeral stream segments. Overall qualitative conditions of soils, such as crusted and erodible soils, were also documented. The survey locations were selected primarily on the basis of accessibility and the allowable time for the field work due to the large extent and restrictions (e.g., construction and permit).

The reference data for algorithm development and validation were obtained by manually digitizing ephemeral stream channels in the VHSR imagery. The ephemeral channel locations mapped during the field work were used to help interpret the images. This approach was taken primarily because some drainage types were difficult to map in the field without synoptic viewing of the landscape, which could introduce a bias in the reference data. An important factor that was considered is that mapping ephemeral streams extensively in desert environments is extremely challenging and expensive. The VHSR imagery provided synoptic viewing and had sufficiently fine spatial resolution for resolving narrow, dry stream channels in the landscape, which supports reliable delineation of ephemeral steam channels across various drainage types.

\subsection{Methods}

The image processing algorithm incorporates information about landscape features and structure that are associated with the surface hydrology of desert regions (Figure 2) via a series of spectral transformation and spatial statistical operations. We observed relatively homogeneous, loose, sandy soil or fine, smooth silt along channel beds in the field. The locations of these surface types corresponded to bright features in the VHSR imagery. Distinct linear vegetation growth that formed riparian corridors along the bright surface strongly corresponded to the locations of ephemeral stream channels. The algorithm was formulated to characterize these landscape features and their spatial patterns in a series of image processing operations in order to map ephemeral stream channels. 
Conceptual Workflow

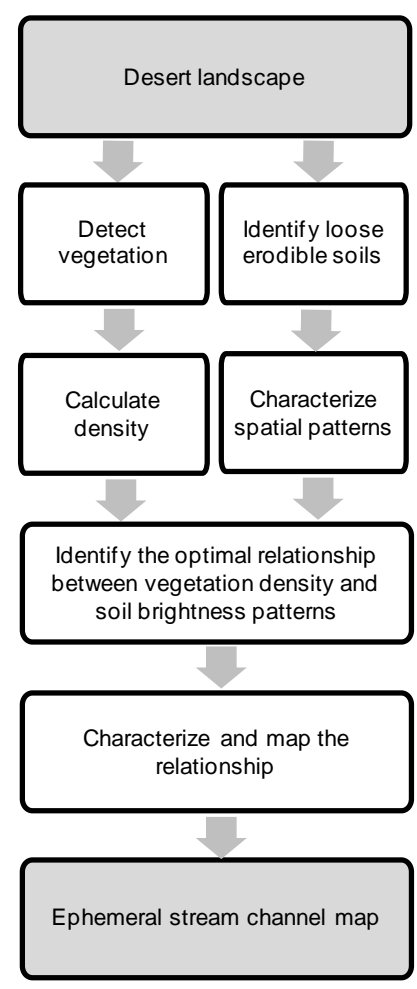

Image Processing Workflow

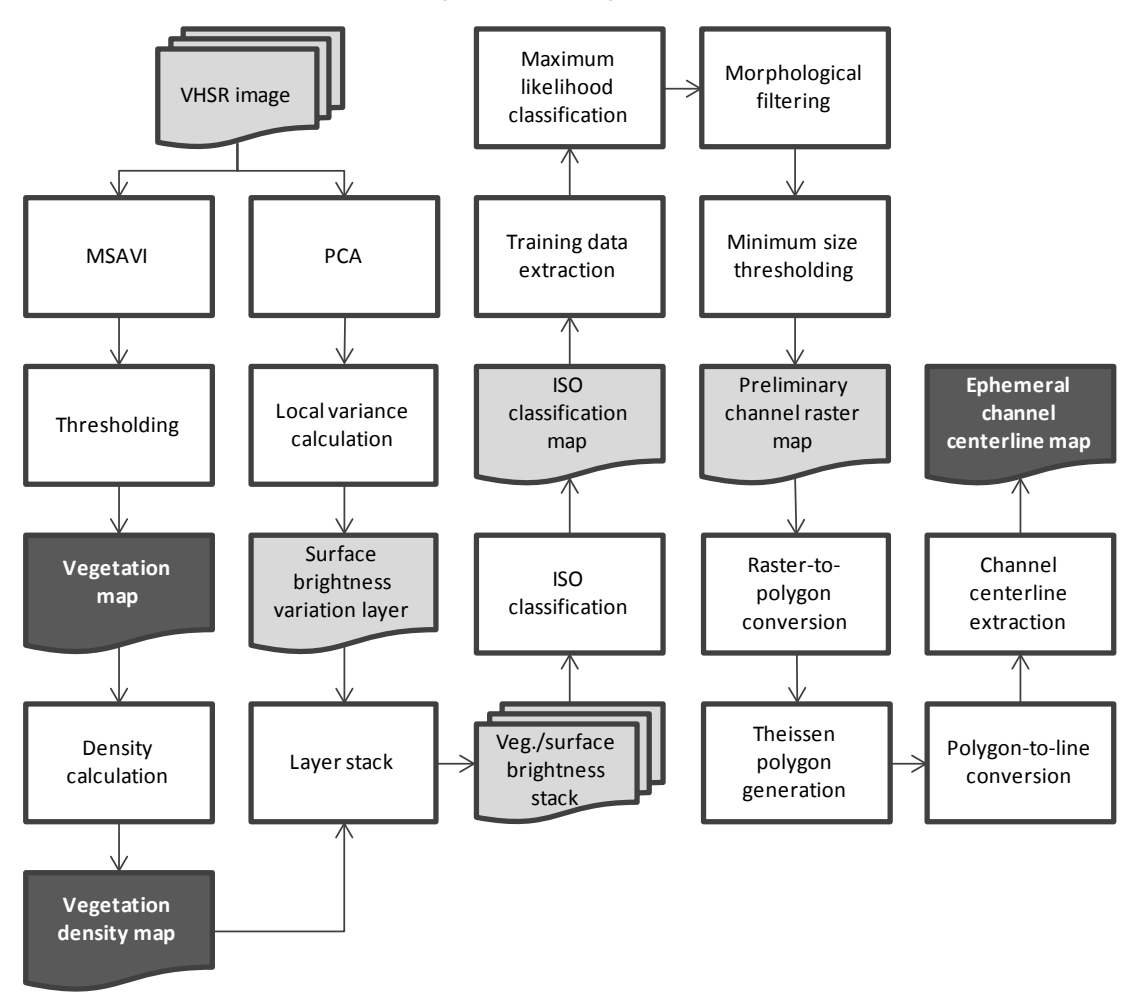

Figure 2. Workflow overview of ephemeral stream channel extraction using very-high-spatialresolution multispectral imagery. (The output and intermediate products that could inform land and resource management decisions are shown in dark grey.) PCA = principal component analysis; ISO = iterative self-organizing.

The VHSR multispectral image mosaic was calibrated to reflectance on the basis of the field spectral reflectance signature from bright, sandy soils in the study area. Agricultural and developed areas were masked out by using the land-use land-cover map from the U.S Geological Survey's (USGS’s) National Gap Analysis Program (Lennartz et al. 2008; Lowry et al. 2005). The modified soil-adjusted vegetation index (MSAVI), which compensates for the effects from the bright soil background of sparsely vegetated areas (Qi et al. 1994), was computed to characterize vegetation. MSAVI was formulated as follows:

$$
M S A V I=\frac{2 \rho_{N I R}+1-\sqrt{\left(2 \rho_{N I R}+1\right)^{2}-8\left(\rho_{N I R}-\rho_{r e d}\right)}}{2}
$$

where $\rho$ is a reflectance value of a spectral band. MSAVI values greater than or equal to 0.14 were determined to be vegetation. This minimum value corresponds to -1.4 standard deviations from the mean MSAVI values of vegetation. The vegetation map included vegetation types that commonly correspond to ephemeral stream channel locations. The density of the vegetation within a 5-m radius was calculated by computing a focal mean within a circular window using the focal analysis tool in ERDAS Imagine 2013 (http://www.hexagongeospatial.com/products/ remote-sensing/erdas-imagine). The vegetation density map helped identify riparian corridors and characteristic vegetation patterns along narrow channels. The 5-m-radius area used for 
calculating the density was interactively determined on the basis of the typical vegetation canopy or patch size and the distances between such features that were observed in the study area.

A principal component analysis (PCA) was performed on the VHSR imagery. The goal of the analysis was to enhance the brightness of the land surface by maximizing the amount of variation in the brightness within the imagery. The local variability of the first principal component layer, which indicates the greatest range of pixel brightness values, was obtained by computing a focal standard deviation within a 1.5-m-radius area. The brightness variability layer enhanced the characteristic brightness heterogeneity that resulted from the complex mixture of loose, erodible soils and adjacent vegetation observed along ephemeral stream channels. The spatial unit for the variability calculation was determined on the basis of the size of typical vegetation canopies and patches as well as the spatial frequency of vegetation and soil occurrences in riparian zones. Although the output from the PCA depends on the spectral variation of the input data, which may affect the repeatability of the algorithm, it is considered useful because spectral characteristics within the study area are expected to be relatively consistent. This assumption is based on the small number of land cover types and relatively small amount of intra-annual vegetation change, which is associated with slow growth in desert environments. In addition, the target of interest in the PCA output is bright soils, which nearly always appear as the brightest features in the first principal component layer. Excluding irrelevant land cover types before processing would prevent the introduction of the significant differences in spectral variation associated with changes in irrelevant parts of the landscape (e.g., developed and agricultural areas) over time.

The vegetation density layer and the layer representing the spatial heterogeneity of the surface brightness were stacked, and the layer stack provided a physical basis for characterizing ephemeral stream channels. An unsupervised classification routine was performed on the layer stack using an iterative self-organizing technique (Tou and Gonzales 1974). It generated 50 classes based on similarities in the vegetation density and surface brightness heterogeneity. Classes that corresponded to ephemeral stream channels were identified in the classification map by comparing it to the reference map. Ephemeral stream channels that covered a range of variability in vegetation density and brightness heterogeneity across the landscape were identified on the basis of visual inspection using the classification map. Statistical properties of vegetation density and brightness heterogeneity were extracted from each of these corresponding classes to obtain comprehensive ephemeral stream channel training data. The training data were used to perform a maximum likelihood classification on the layer stack in order to generate a preliminary ephemeral stream channel map.

A morphological closing operation with a 1.5-m-radius kernel was applied to the preliminary map to fill in missing pixels of ephemeral stream channel and to remove isolated fragments. Other aims of this operation were to account for spatial autocorrelation and reduce artifacts that were likely introduced by noise in the original image data. A cluster of candidate channel pixels that was composed of less than 30,000 pixels (approximately $675 \mathrm{~m}^{2}$ ) was eliminated to exclude spatially incohesive clusters, and the final ephemeral channel classification map was generated. The raster processing of the algorithm was performed in the ERDAS Imagine. 
The groups of pixels that represent ephemeral stream channels in the classification map were converted to polygons using the raster-to-polygon tool in ArcGIS 10.2 (Figure 3a). Edges of the channel polygons were smoothed, and small holes in the channel polygons, which were introduced during the conversion process, were removed by applying a $50-\mathrm{m}^{2}$ minimum-size filter. The channel polygons were further converted to points, and additional vertices were generated every $5 \mathrm{~m}$ along the polygon edges (Figure 3b). Thiessen polygons were then created around the channel edge vertices, creating a network of lines; some of these lines represent the channel centerlines (Figure 3c). Among the Thiessen polygons, edges corresponding to channels were identified, and centerlines of the channels, which were represented as one edge of the polygons, were extracted (Figure 3d). Irrelevant lines connecting to the centerlines were removed to finalize the ephemeral channel centerline map (Figures 3e and 3f). The vector processing of the algorithm was developed in Python in the Esri ArcGIS software (http://www.arcgis.com).

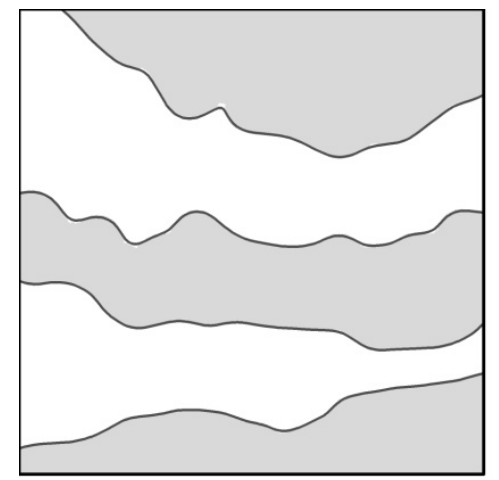

(a)

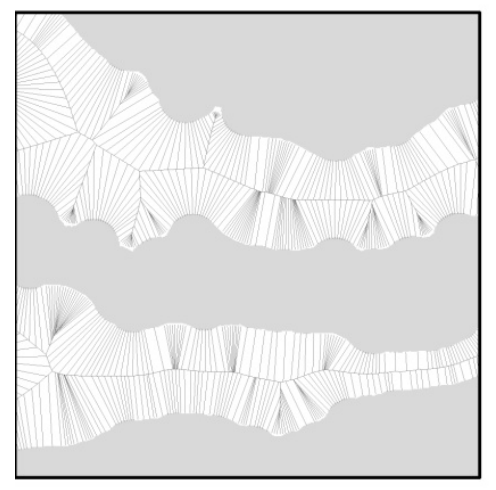

(d)

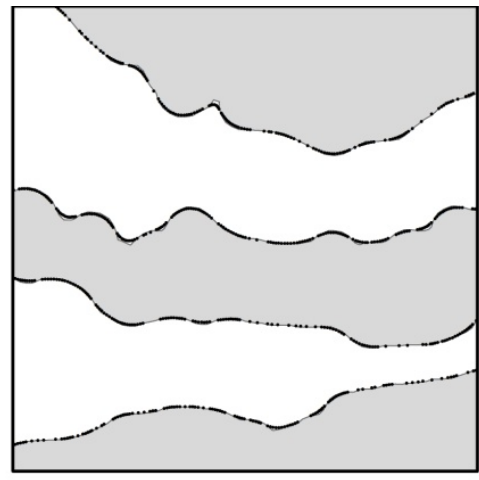

(b)

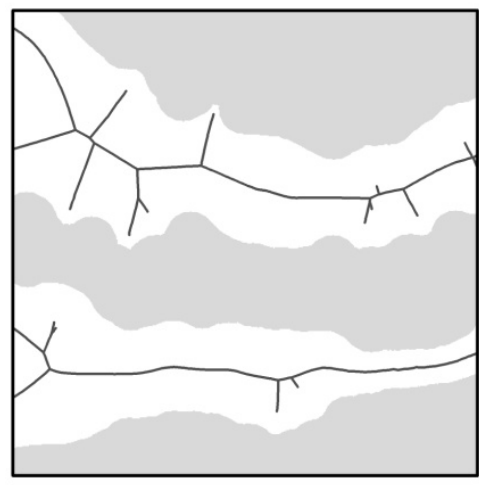

(e)

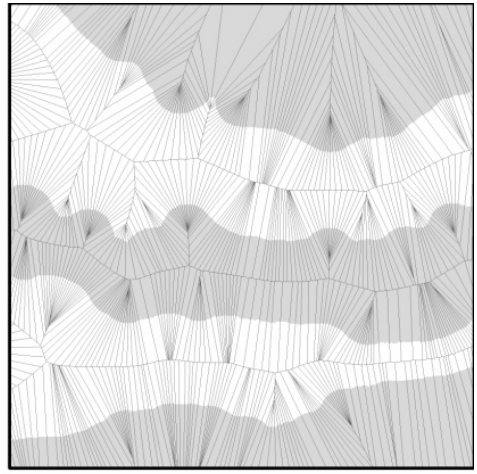

(c)

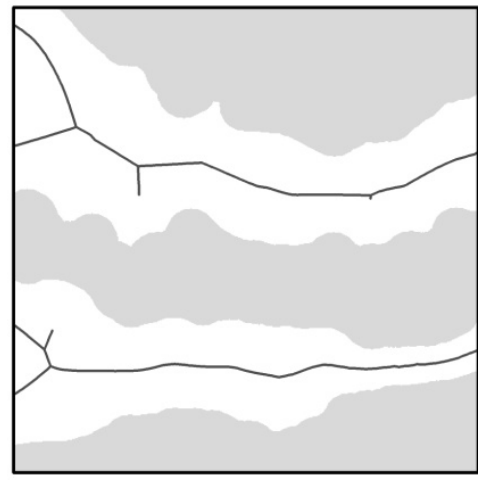

(f)

Figure 3. Procedure of channel centerline extraction using the raster channel map. (a) Channel polygons converted from the raster map, (b) points corresponding to channel edges, (c) Thiessen polygons generated around the points, (d) Thiessen polygons trimmed to channels, (e) polylines extracted from the Thiessen polygons, and (f) final channel centerlines.

Both ephemeral channel classification and centerline maps were examined to evaluate how well the algorithm extracts stream channels in desert landscapes. The channel classification map was first qualitatively examined with regard to the overall abundance and spatial patterns of the extracted channels by comparing it with the NHD (USGS 2008). Quantitative analysis was 
performed by using two 6- $\mathrm{km}^{2}$ areas referred to as the east and west assessment areas. These assessment areas were selected to contain a range of stream types across the landscape. In each assessment area, 10,000 pixels that were located at least $1 \mathrm{~m}$ (7 pixels) away from each other were randomly selected using the ArcGIS software. Pixels located within $1 \mathrm{~m}$ of channel boundaries were excluded from the assessment to compensate for uncertainty in the positional accuracy of reference polygon boundaries. The remaining validation pixels were used to compute accuracy metrics. Accuracy metrics were calculated using the accuracy assessment template developed in our preliminary study using Microsoft Excel (https://products.office.com/en-us/excel).

To evaluate the channel centerline map, the abundance and spatial distributions of channels were first qualitatively compared with the coarse-scale NHD to examine the level of detail that the aerial remote sensing product represents. The local-scale accuracy of channel centerlines was evaluated on the basis of the proportion of the length of the extracted channel to the length of the corresponding reference channel (i.e., 100\% delineation, meaning that extracted channel length equals reference channel length, represented the highest accuracy). The analysis included all channel segments longer than $150 \mathrm{~m}$ in both assessment areas. Then two criteria, $\geq 70 \%$ and $\geq 50 \%$, were applied. The number of reference channels with $\geq 70 \%$ and $\geq 50 \%$ of their channel delineated by the algorithm were identified and examined by an independent analyst in order to determine whether the delineated channel centerlines could be visually recognized as channels regardless of the proportion delineated. To minimize bias, the independent analyst was not involved in developing the algorithm or generating the reference data. This qualitative method was employed in order to gain an understanding of the spatial patterns of extracted channel segments.

\section{Results and Discussion}

The spectral index and transformation layers produced within the algorithm are shown in Figure 4. In the subset layers, the ephemeral stream channels mostly drain from left to right (i.e., from west to east; Figure 4a). Loose, sandy soil and fine silt appear as a white to light grey tone in the first principal component layer, which includes both channel beds and surfaces covered with bare ground and sparse vegetation (Figure 4b). These indistinguishable land cover types appear to have distinct spatial heterogeneity in their brightness, which is depicted in the standard deviation of the first principal component (Figure 4c). Vegetation canopies and patches exhibit high MSAVI values (Figure 4d), and applying a minimum threshold value successfully produces a vegetation map (Figure 4e). The vegetation density effectively filters scattered vegetation and accentuates riparian corridors and linear vegetation growth along ephemeral stream channels (Figure 4f). The spatial heterogeneity of the surface brightness (Figure 4c) and vegetation density (Figure 4f) strongly indicates the presence of ephemeral stream channels. The output map captures areas having the characteristics of ephemeral streams in desert environments, such as dense linear vegetation growth and bright sandy soils (Figure 4g). Channel centerlines that were extracted from the map show a strong similarity to the distributions of ephemeral stream channels in the reference map (Figures $4 \mathrm{~h}$ and $4 \mathrm{i}$ ). 
When compared to the reference channel map of the assessment areas, the raster map (Figure 4g) has a few disconnected channel segments where vegetation densities and brightness heterogeneities are slightly outside the range defined by the training data. Some headwaters where vegetation growth was no longer dense enough to exhibit linear features were also not detected (Figure 3a, 3f, and 3h).

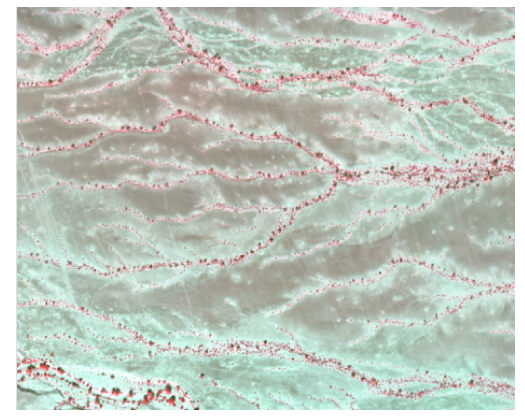

(a)

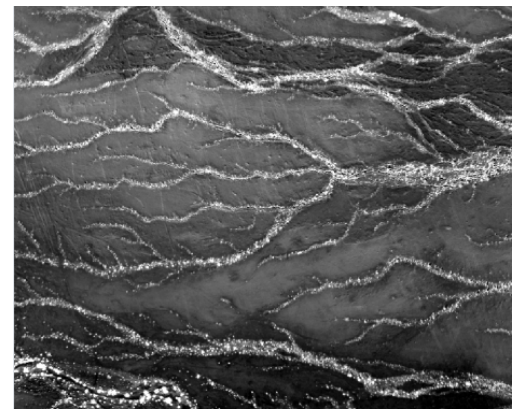

(d)

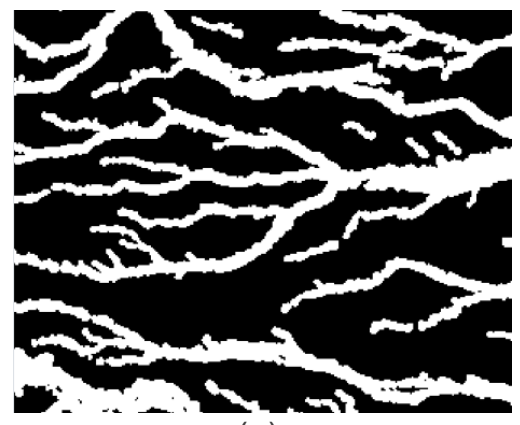

(g)

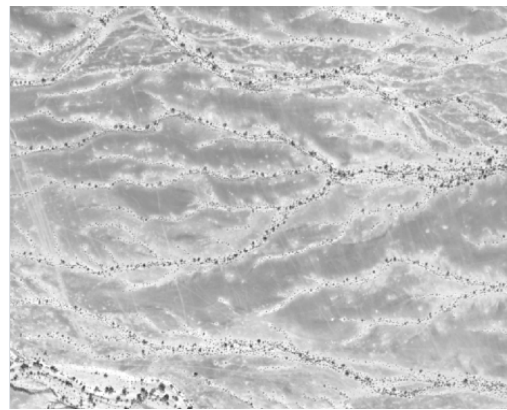

(b)

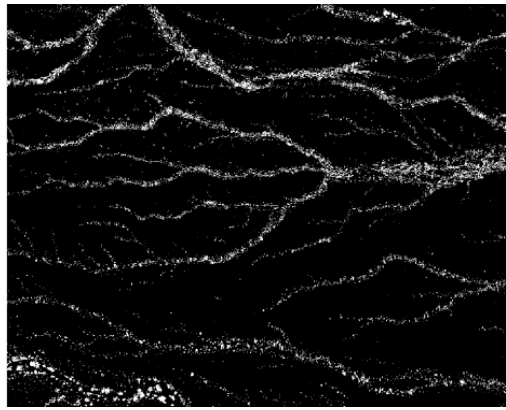

(e)

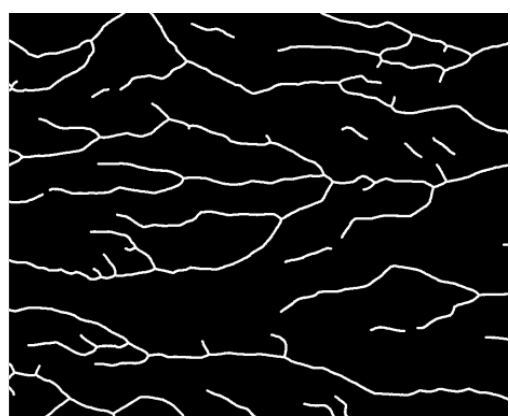

(h)

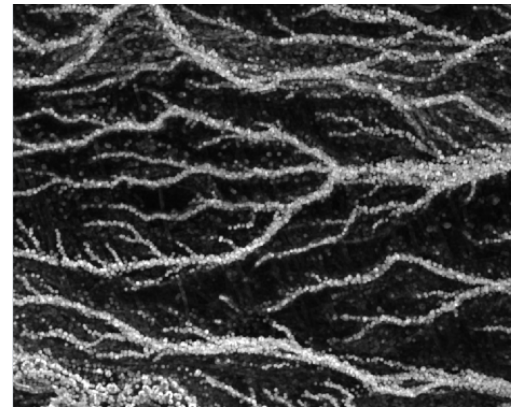

(c)

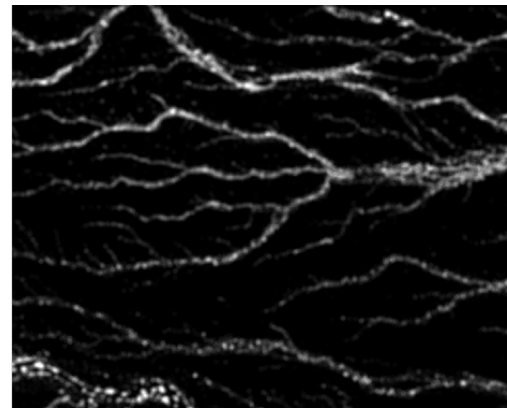

(f)

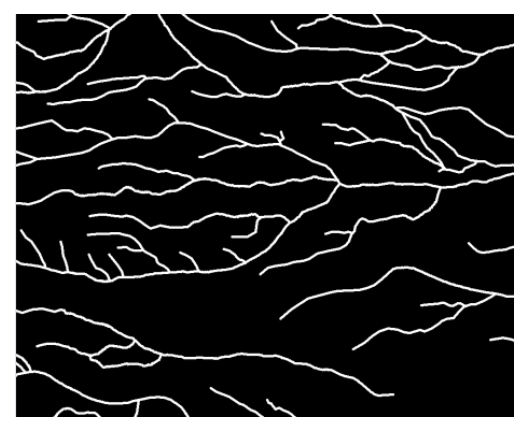

(i)

Figure 4 Close-up views of the input image and spectral index and transformation layers within the west assessment area. (a) 15-cm-resolution multispectral imagery displayed in false color; (b) principal component 1; (c) spatial heterogeneity of principal component 1 within the 1.5-mradius area; (d) modified soil-adjusted vegetation index; (e) occurrence of vegetation; (f) vegetation density within the 5-m-radius area; (g) ephemeral stream channel areas; (h) final ephemeral stream centerlines; and (i) reference centerline delineated on the basis of image interpretation of the 15-cm-resolution multispectral imagery. 
When examining ephemeral stream channel networks at a landscape scale, the algorithm identified a large number of narrow channels across the study area (Figure 5). The channel classification map indicated the accurate identification of McCoy Wash, the largest stream feature in the study area (see Figure 1). Numerous narrow tributaries occur along the hill slope on both sides of the wash. The algorithm captured a range of channel widths and forms in both the west and the east assessment areas. Underclassification (or false negatives) was often observed near stream headwaters and areas characterized by braided channel networks; overclassification (or false positives) often occurred around wide channels.

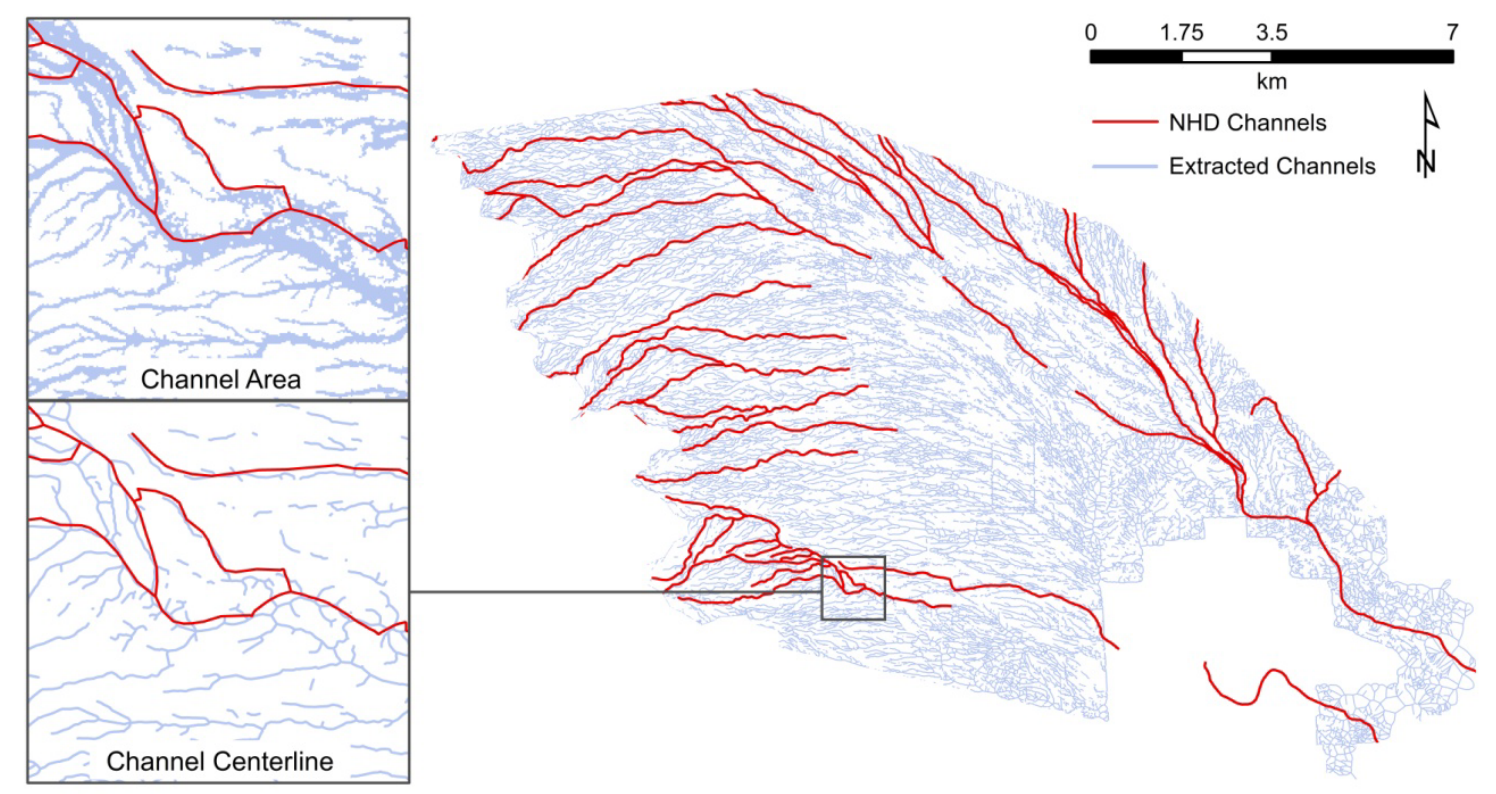

Figure 5. Maps of ephemeral stream channels derived from the very-high-spatial-resolution image overlain with the NHD. Close views show the raster channel map representing ephemeral stream beds (upper left) and the vector map representing the channel centerlines (lower left).

Accuracy ranged from $48.5 \%$ to $91.8 \%$, depending on the specific accuracy measures (Table 1). The majority of accuracy measures exceeded $75 \%$ across the assessment areas. The producer's accuracy was higher than the user's accuracy in both areas, which indicated falsepositive detection, or overclassification, in the ephemeral channel map. This overclassification was intentionally created during the raster processing as a part of the strategy for increasing the connectivity of ephemeral stream channels during the channel centerline extraction. Kappa coefficients (0.54-0.61) suggested that the classification accuracy was generally better than random. 
Table 1 Accuracy of Ephemeral Stream Channel Maps Derived from Remotely Sensed Imagery

\begin{tabular}{lccc}
\hline Classification Accuracy & Pooled & West & East \\
\hline Overall accuracy (\%) & 77.0 & 91.1 & 79.8 \\
Producer's accuracy (\%) & 85.8 & 91.8 & 88.2 \\
User's accuracy (\%) & 52.2 & 48.5 & 51.4 \\
Kappa coefficient & 0.55 & 0.61 & 0.54 \\
\hline & & & \\
\hline Centerline Extraction Accuracy & Pooled & West & East \\
& & & \\
\hline Total channel length (km) & 146.0 & 50.9 & 95.1 \\
& $(154.0)^{*}$ & $(66.3)$ & $(87.7)$ \\
Accurate channel area (km²) & 2.0 & 0.8 & 1.2 \\
& $(2.7)$ & $(1.3)$ & $(1.5)$ \\
Accurate channel length (km) & 92.3 & 37.7 & 54.6 \\
& $(153.7)$ & $(66.0)$ & $(87.7)$ \\
Channel density (km/km²) & 8.5 & 6.4 & 9.7 \\
& $(13.3)$ & $(11.2)$ & $(15.3)$ \\
Average channel width (m) & 33.7 & 19.7 & 47.6 \\
& $(32.0)$ & $(18.1)$ & $(45.9)$ \\
Accurate delineation $\geq 70 \%(\%)$ & 56.3 & 53.0 & 59.5 \\
Accurate delineation $\geq 50 \%(\%)$ & 66.5 & 65.5 & 67.5 \\
Recognized (\%) & 89.0 & 86.0 & 92.0 \\
\hline
\end{tabular}

${ }^{*}$ Values in parentheses indicate reference data.

A considerable number of channel centerlines were delineated by the algorithm across the study area (Figure 5), and the channel centerlines of McCoy Wash and other major tributaries were correctly delineated (Figure 5). Minor or narrow channels and channels occurring in areas with even, sparse vegetation growth were frequently undetected. Channel features near stream headwaters were often missing from the final channel map. Although the algorithm correctly delineated wide channels consisting of well-defined braided forms, it had difficulty in delineating wide channels with single-threaded forms when they contained heterogeneous vegetation growth.

The difference between channel length delineated by the algorithm and the corresponding reference channel length varied depending on the assessment area (i.e., $22 \%$ and $8 \%$ for the east and west assessment areas, respectively) (Table 1). While the extracted channel areas were generally similar to the reference channel areas, approximately $40 \%$ fewer channels were extracted compared with the reference data. This considerable discrepancy in channel length was mostly explained by the differences between the reference data and algorithm in how channels were defined. For example, a wide tributary consisting of braided channels separated by a small distance may be delineated as multiple channels in the reference data, but may be identified as a single broad channel by the algorithm. Surface patterns across desert scrublands that were created by unstructured surface flow or sheet-flow may be defined as channels in the reference data but may not be recognized as channels by the algorithm because of the lack of typical characteristics of ephemeral channels. Establishing a definition of ephemeral stream channels that is meaningful from ecological and management perspectives (e.g., active versus inactive, a single aggregated tributary versus a set of individual braided channels) is needed in order to 
generate reliable reference data and to refine the algorithm. This definition would help provide valuable detailed distributions of ephemeral stream channels for local or landscape-scale hydrological modeling and inform resource management decisions.

Within the two assessment areas, $\geq 70 \%$ and $\geq 50 \%$ of the channel lengths were delineated for more than one-half and two-thirds of the reference channel segments, respectively (Table 1). More than 53\% and $65.5 \%$ of the reference channels were accurately delineated over $\geq 70 \%$ and $\geq 50 \%$ of the reference channel length, respectively. The accuracies in the channel centerline delineation for the west and east areas were nearly identical. Despite the modest accuracy of the criteria-based assessment, which is defined by the percentage of the length of the delineated channel, an analyst determined that about $90 \%$ of the reference segments were correctly extracted on the basis of the combination of the delineated channel lengths in each segment and the distribution or delineated line fragments within a validation segment. For example, when $40 \%$ of the channel length for a reference segment was delineated by the algorithm, the segment detection was not considered a success on the basis of the $70 \%$ or $50 \%$ criterion. However, it might have been considered a success in the analyst-based assessment, when the analyst recognized the segment from the delineated centerlines, which might consist of small channel fragments linearly distributed across the validation segment. This high recognition rate suggests that the channel segments extracted by using the algorithm were spatially cohesive and had a strong association with ephemeral stream channels present in the study area. This result indicates that including a pattern recognition routine in the algorithm could further improve channel extraction accuracy.

\section{Conclusions}

The algorithm extracted detailed ephemeral stream channels in a desert landscape, and provided significantly greater channel delineation than the NHD. The overall accuracy of channel classification was $77 \%$, and the accuracy of delineation ranged from $53 \%$ to $92 \%$ depending on the accuracy criterion. Tributaries associated with well-defined riparian corridors and tributaries with strong contrast from surrounding areas were accurately classified and delineated by the algorithm. The limited success of the algorithm in mapping stream headwaters, which often occupy a small portion of total channel length, can be explained by their unique surface characteristics. These dry stream headwaters are linear features that are characterized by bright, loose soils with minimal vegetation growth; these characteristics are very different from those typical of downstream channels in the landscape. While areas that contained desert pavement exhibited strong spectral contrasts between stream channels and uplands, downstream channels that consisted of bright soils with sparse vegetation obscured the target-background contrast, and as a result, some channels were undetected.

The heterogeneous nature of channel forms in ephemeral stream networks influenced the accuracy of channel classification and centerline delineation. Wide tributaries characterized by well-defined, braided channels with linear vegetation growth were accurately classified and delineated. In contrast, the channels characterized by varying vegetation cover or weakly developed riparian corridors were often mistaken for a set of braided channels, creating a discrepancy with the reference data. 
While further improvement is warranted, the algorithm showed the capability of mapping detailed ephemeral stream networks in a desert landscape across various channel sizes and forms by using VHSR multispectral imagery. The algorithm provides a means of obtaining detailed information about surface hydrologic features in desert regions-information that is difficult to obtain at the watershed scale. Thus, it has significant potential for supporting analyses and models of hydrologic and sediment transport processes that pertain to the critical functionality of ephemeral streams, which are currently limited by the coarse scale of hydrologic data.

This study demonstrated the effectiveness of the systematic integration of landscape features and properties (e.g., vegetation and surface brightness) and structures (e.g., abundance and spatial patterns) into an image-processing algorithm for mapping ephemeral stream channels in desert environments. Although advanced, complex methods of landscape feature extraction exist, we chose a simple, intuitive, and tractable approach and formulated the algorithm by logically linking landscape information associated with surface hydrology. In addition to providing a detailed map of ephemeral stream networks, this tractable algorithm allows ecologists and resource managers to extract information about land resources from intermediate products generated within the algorithm, such as maps of the distribution and density of vegetation. For example, a vegetation map can be used to compute vegetation cover within a unit area, and this result can provide valuable information for estimating surface erosion or examining vegetation community state. As indicated in this study, a vegetation density map would be useful for identifying riparian corridors in desert regions. A vegetation density map could provide insights into the condition of wildlife species' habitats by indicating the availability of potential shelters and food. Such a result suggests the broad applicability of this algorithm. The framework is also transferable to other physiognomically comparable arid environments.

Currently, we are testing the robustness of the ephemeral channel extraction algorithm by using VHSR images collected on January 2014 from the comparable area. The images have considerable forward and side overlaps (e.g., >70\%), which facilitates the generation of a digital surface model. The possibility of integrating image-based height information into the algorithm is also being considered as part of our current effort.

The algorithm developed in this study used a single-date image mosaic with minimal preprocessing in order to preserve the integrity of raw spectral signals in the data; thus, it minimized any uncertainty in its performance that would be associated with data manipulation during preprocessing. The algorithm should be tested for its robustness by using imagery from different dates or different areas. Before the algorithm is applied for environmental monitoring practice, a rigorous assessment of its accuracy and a sensitivity analysis are necessary to determine how much and what types of data preparation (e.g., rectification, calibration, registration) are required for multi-temporal image sets in order to reliably detect and quantify changes in target features and properties.

Ephemeral stream channels in desert regions are tremendously heterogeneous. The channels exhibit a range from well-defined channels along riparian corridors to obscured channels that are nearly indistinguishable from surrounding land cover types within expansive 
washes. In order to produce truly meaningful ephemeral stream channel maps for hydrological modelling and resource management, a standardized definition of ephemeral stream channels in desert regions needs to be established. The definition can be based on properties of channels that can be used to differentiate active channels from inactive channels.

When the additional research and investigations suggested here are conducted, the ephemeral stream channel extraction algorithm formulated in this study will be more useful in facilitating the development of cost-effective, long-term monitoring strategies and the establishment of guidelines and regulatory protection for ephemeral streams. The potential for providing information about multiple resources suggests the extended utility of the algorithm for other natural resources. Contributions to establishing of ephemeral stream monitoring guidelines and providing multiple-resource data would aid sustainable utility-scale solar energy development in desert regions.

\section{Acknowledgments}

The authors thank Katherine E. Rollins and Scott O. Schlueter for their qualitative accuracy assessment. They also thank Karen P. Smith, Mark A. Grippo, and Esther E. Bowen at Argonne National Laboratory, anonymous reviewers involved in the U.S. Department of Energy's (DOE's) SunShot Initiative, and subject matter experts for insightful comments on the manuscript. The submitted manuscript has been created by UChicago Argonne, LLC, Operator of Argonne National Laboratory ("Argonne”). Argonne, a DOE Office of Science laboratory, is operated under Contract No. DE-AC02-06CH11357. The U.S. Government retains for itself, and others acting on its behalf, a paid-up nonexclusive, irrevocable worldwide license in said article to reproduce, prepare derivative works, distribute copies to the public, and perform publicly and display publicly, by or on behalf of the Government. The project was funded by DOE's SunShot Initiative (\#27239).

\section{References}

Abella, S.R., 2010. Disturbance and plant succession in Mojave and Sonoran Deserts of the American Southwest. International Journal of Environmental Research and Public Health 7, 1248-1284.

Belnap, J., 1995. Surface disturbances: Their role in accelerating desertification. Environmental Monitoring and Assessment 37, 39-57.

BLM (Bureau of Land Management), 2015. Solar Energy. http://www.blm.gov/wo/st/en/prog/energy/solar_energy.html.

BLM and DOE (U.S. Department of Energy), 2012. Final Programmatic Environmental Impact Statement (PEIS) for Solar Energy Development in Six Southwestern States. FES 12-24, DOE/EIS-0403. Washington, DC. 
Bull, L.J., and M.J. Kirkby (editors), 2002. "Dryland River Characteristics and Concepts," Chap. 1 in Dryland Rivers: Hydrology and Geomorphology of Semi-Arid Channels, John Wiley and Sons, Ltd., Chichester, England.

Caruso, B.S., and J. Haynes, 2011. Biophysical-regulatory classification and profiling of streams across management units and ecoregions. Journal of the American Water Resources Association 47, 386-407.

Chin, A., and K.J. Gregory, 2001. Urbanization and adjustment of ephemeral stream channels. Annals of the Association of American Geographers 91(4), 595-608.

Cho, H.C., K.C. Slatton, C.R. Krekeler, and S. Cheung, 2011. Morphology-based approaches for detecting stream channels from ALSM data. International Journal of Remote Sensing 32, 9571-9597.

Duniway, M.C., and J.E. Herrick, 2011. Disentangling road network impacts: The need for a holistic approach. Journal of Soil and Water Conservation 66, 31A-36A.

EPA (U.S. Environmental Protection Agency), 2014. Automated Geospatial Watershed Assessment Tool. http://www.epa.gov/esd/land-sci/agwa/.

Field, J.J., and R.W. Lichvar (editors), 2007. Review and Synopsis of Natural and Human Controls on Fluvial Channel Processes in the Arid West. ERDC/CRREL TR-07-16. U.S. Army Corps of Engineers, Engineer Research and Development Center, Cold Regions Research and Engineering Laboratory, Hanover, NH.

Fisher, S.G., 2006. "Stream Ecosystems of the Western United States," in River and Stream Ecosystems of the World, C.E. Cushing, K.W. Cummins, and G.W. Minshall (editors), University of California Press, Oakland, CA.

Fritz, K.M., E. Hagenbuch, E. D’Amico, M. Reif, P.J. Wigington Jr., S.G. Leibowitz, R.L. Comeleo, J.L. Ebersole, and T.L. Nadeau, 2013. Comparing the extent and permanence of headwater streams from two field surveys to values from hydrographic databases and maps. Journal of the American Water Resources Association 49, 867-882.

Hamada, Y., D.A. Stow, D.A. Roberts, F. Franklin, and P.C. Kyriakidis, 2013. Assessing and monitoring semi-arid shrublands using object-based image analysis and multiple endmember spectral mixture analysis. Environmental Monitoring and Assessment 185(4), 3173-3190. doi: 0.1007/s10661-012-2781-z.

Jacobson, P.J., and K.M. Jacobson, 2013. Hydrologic controls of physical and ecological processes in Namib Desert ephemeral rivers: Implications for conservation and management. Journal of Arid Environments 93, 80-93.

Jenson, S.K., 1991. Application of hydrologic Information automatically extracted from digital elevation models. Hydrological Processes 5, 31-44. 
Lashermes, B., E. Foufoula-Georgiou, and W.E. Dietrich, 2007. Channel network extraction from high resolution topography using wavelets. Geophysical Research Letters 34(23), L23S04. doi: 10.1029/2007GL031140.

Lennartz, S., T. Bax, J. Aycrigg, A. Davidson, M. Reid, and R. Congalton, 2008. Final Report on Land Cover Mapping Methods for California Map Zones 3, 4, 5, 6, 12, and 13. National Biological Information Infrastructure, ID.

Levick, L., J. Fonseca, D. Goodrich, M. Hernandez, D. Semmens, J. Stromberg, R. Leidy, M. Scianni, D.P. Guertin, M. Tluczek, and W. Kepner, 2008. The Ecological and Hydrological Significance of Ephemeral and Intermittent Streams in the Arid and Semiarid American Southwest, EPA/600/R-08/134, ARS/233046, U.S. Environmental Protection Agency and USDA/ARS Southwest Watershed Research Center, Tucson, AZ., 116 pp.

Li, J., and D.W.S. Wang, 2010. Effects of DEM sources on hydrologic applications. Computers, Environment and Urban Systems 34(3), 251-261.

doi: 10.101/j.compenvurbsys.2009.11.002.

Lowry, J.H., Jr., R.D. Ramsey, K. Boykin, D. Bradford, P. Comer, S. Falzarano, and B. Wolk, 2005. Southwest Regional Gap Analysis Project: Final Report on Land Cover Mapping Methods, RIS/GS Laboratory, Utah State University, Logan, UT.

Passalacqua, P., P. Belmont, and E. Foufoula-Georgiou, 2012. Automatic geomorphic feature extraction from LiDAR in flat and engineered landscapes. Water Resources Research 48(3), W03528. doi: 10.1029/2011WR010958.

Pool, D.R., 2005. Variations in climate and ephemeral channel recharge in southeastern Arizona, United States. Water Resources Research 41(11), W11403.

Qi, J., A. Chehbouni, A.R. Huete, and Y.H. Kerr, 1994. Modified soil adjusted vegetation index (MSAVI). Remote Sensing of Environment 48(2), 119-126. doi: 10.1016/0034-4257(94)90134-1.

Sant, E.D., G.E. Simonds, R.D. Ramsey, and R.T. Larsen, 2014. Assessment of sagebrush cover using remote sensing at multiple spatial and temporal scales. Ecological Indicators 43, 297-305.

Steward, A.L., D. von Schiller, K. Tockner, J.C. Marshall, and S.E. Bunn, 2012. When the river runs dry: Human and ecological values of dry riverbeds. Frontiers in Ecology and the Environment 10, 202-209. doi: 10.1890/110136.

Tooth, S., 2000. Process, form and change in dryland rivers: A review of recent research. EarthScience Reviews 51(1-4), 67-107. 
Tou, J.T., and R.C. Gonzalez, 1974. Pattern Recognition Principles. Addison-Wesley Publishing Company, Ontario-Sydney-Tokyo.

USGS (U.S. Geological Survey), 2008. National Hydrography Dataset. http://nhd.usgs.gov. Accessed on January 10, 2013

Vyverberg, K., 2010. A Review of Stream Processes and Forms in Dryland Watersheds. California Department of Fish and Game, Sacramento, CA.

Wang, H., Y. Shao, and L.M. Kennedy, 2014. Temporal generalization of sub-pixel vegetation mapping with multiple machine learning and atmospheric correction algorithms. International Journal of Remote Sensing 35(20), 7118-7135.

Witztum, E.R., and D.A. Stow, 2004. Analyzing direct impacts of recreation activity on coastal sage scrub habitat with very high resolution multi-spectral imagery. International Journal of Remote Sensing 25, 3477-3496.

Wohl, E., D. Cooper, L. Poff, F. Rahel, D. Staley, and D. Winters, 2007. Assessment of stream ecosystem function and sensitivity in the Bighorn National Forest, Wyoming. Environmental Management 40, 284-302.

Yang, K., and L.C. Smith, 2013. Supraglacial streams on the Greenland Ice Sheet delineated from combined spectral-shape information in high-resolution satellite imagery. IEEE Geoscience and Remote Sensing Letter 10(4), 801-805.

Yang, K., M. Li, Y. Liu, L. Cheng, Y. Duan, and M. Zhou, 2014. River delineation from remotely sensed imagery using a multi-scale classification approach (online). IEEE Journal of Selected Topics in Applied Earth Observations and Remote Sensing. http://ieeexplore.ieee.org/stamp/stamp.jsp?arnumber=6777572. Accessed on June 18, 2014. 